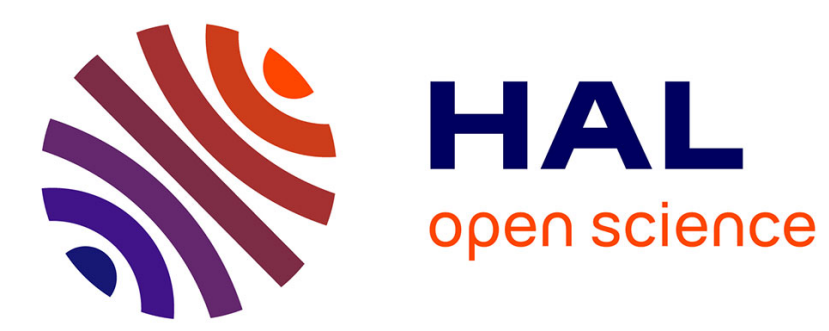

\title{
Hadronic B decays at BABAR
}

A. Zghiche

\section{To cite this version:}

A. Zghiche. Hadronic B decays at BABAR. QCD 05 12th High Energy Physics International Conference in Quantum Chromodynamics, Jul 2005, Montpellier, France. pp.185-188, 10.1016/j.nuclphysbps.2006.11.084 . in2p3-00024908

\section{HAL Id: in2p3-00024908 https://hal.in2p3.fr/in2p3-00024908}

Submitted on 18 Oct 2005

HAL is a multi-disciplinary open access archive for the deposit and dissemination of scientific research documents, whether they are published or not. The documents may come from teaching and research institutions in France or abroad, or from public or private research centers.
L'archive ouverte pluridisciplinaire HAL, est destinée au dépôt et à la diffusion de documents scientifiques de niveau recherche, publiés ou non, émanant des établissements d'enseignement et de recherche français ou étrangers, des laboratoires publics ou privés. 


\title{
Hadronic B decays at BABAR
}

\author{
A. Zghiche \\ for the BABAR Collaboration \\ LAPP-IN2P3-CNRS \\ BP. 110, F-74941 Annecy-le-Vieux Cedex
}

\section{Presented at QCD 05}

12th High Energy Physics International Conference in Quantum Chromodynamics Montpellier (France), July 4-8, 2005 


\title{
HADRONIC $B$ DECAYS AT BABAR
}

\author{
A. Zghiche for the BABAR collaboration ${ }^{\mathrm{a}}$

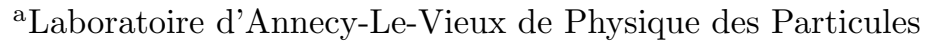 \\ 9 Chemin de Bellevue, BP 110, F-74941 Annecy-le-Vieux CEDEX - France
}

By means of hadronic $B$ decays, the BABAR experiment aims to constrain the CKM matrix performing $C P$ parameter measurements. It also seeks to test QCD factorization predictions and other models for $B$ structure and decay mechanisms. We will present some of the on-going $C P$ related analyses in the first section, while the second section will be dedicated to report on the conducted investigations on subjects as diverse as probing the gluon component in the $B$ meson wave function, new physics and final state interactions in annihilation processes, intrinsic charm searches and first observation of strange charmed baryon production in $B$ decays.

\section{1. $C P$ related analyses}

In this section we report on on-going hadronic $B$ decays measurements of branching fractions and $C P$ asymmetries which are defined, for the $B \rightarrow f$ decay, as :

$$
\mathcal{A}_{C P}=\frac{\mathcal{B}(B \rightarrow f)-\mathcal{B}(\bar{B} \rightarrow \bar{f})}{\mathcal{B}(B \rightarrow f)+\mathcal{B}(\bar{B} \rightarrow \bar{f})}
$$

These are the first steps in analyses that could be used, in the future, to measure CKM matrix parameters like $\gamma \equiv \arg \left[-\frac{V_{u d} V_{u b}^{*}}{V_{c d} V_{c b}^{*}}\right]$ or a combination of $\gamma$ and $\beta \equiv \arg \left[-\frac{V_{c d} V_{c b}^{*}}{V_{t d} V_{t b}^{*}}\right]$.

\subsection{Measurement of the branching frac- tion and decay rate asymmetry of $B^{-} \rightarrow D_{\pi^{+} \pi^{-} \pi^{0}} K^{-}$}

The decays $B \rightarrow D^{(*) 0} K^{(*)}$ can be used to measure the angle $\gamma$ taking advantage of the interference between $b \rightarrow u \bar{c} s$ and $b \rightarrow c \bar{u} s$ decay amplitudes. Different approaches have been developed, among which $\gamma$ measurements involving $D$ decays to multi-body, using a Dalitz plot analysis technique as described in reference [1]. In this analysis, we measure the branching fraction of the decay modes $B^{-} \rightarrow D^{0}\left(\bar{D}^{0}\right) K^{-}$with the $D^{0}\left(\bar{D}^{0}\right)$-decay : $D^{0}\left(\bar{D}^{0}\right) \rightarrow \pi^{+} \pi^{-} \pi^{0}$, which is Cabibbo suppressed. They yield a much smaller event sample compared to Cabibbo allowed $D$ decays but the interfering $D^{0}$ and $\bar{D}^{0}$ amplitudes have similar magnitudes. Therefore, the sensitivity to $\gamma$ of this $D$ decay channel is expected to be relevant. In addition, due to interference, the production rate may differ from the product $\mathcal{B}_{\text {prod }} \equiv$ $\mathcal{B}\left(B^{-} \rightarrow D^{0} K^{-}\right) \times \mathcal{B}\left(D^{0} \rightarrow \pi^{+} \pi^{-} \pi^{0}\right)=(4.1 \pm$ $1.6) \times 10^{-6}$ by up to about $0.2 \mathcal{B}_{\text {prod }}[2]$. From a sample of 229 million of $B \bar{B}$ pairs, we found $133 \pm 23$ signal events which correspond to a branching ratio of $\mathcal{B}\left(B^{-} \rightarrow D_{\pi^{+} \pi^{-} \pi^{0}} K^{-}\right)=$ $(5.5 \pm 1.0 \pm 0.7) \times 10^{-6}$. We determine the raw asymmetry and do not find any significant deviation from zero : $\mathcal{A}_{C P}^{\text {raw }}=0.02 \pm 0.16 \pm 0.03$. The $\gamma$ extraction is underway using the full Dalitz analysis of the $D$-decay [3].

\subsection{Measurement of the branching frac- tion $B^{0} \rightarrow \bar{D}^{0}\left(D^{0}\right) K^{+} \pi^{-}$}

To determine the feasibility of measuring $\gamma$ with the method proposed by R. Aleksan et al. [4], that uses three-body $B \rightarrow D K \pi$ decays, we have studied $\bar{D}^{0}\left(D^{0}\right) K^{+} \pi^{-}$final states with $205 \mathrm{fb}^{-1}$ data sample. In these modes, the CKM suppressed $b \rightarrow u \bar{c} s$ processes contain color allowed diagrams, resulting in larger rates and more significant $C P$ violation effects than the twobody $B \rightarrow D K$ decays. We measured $\mathcal{B}\left(B^{0} \rightarrow\right.$ $\left.\bar{D}^{0} K^{+} \pi^{-}\right)=(8.6 \pm 1.5 \pm 1.0) \times 10^{-5}$ combining $D$ modes $\left(D^{0} \rightarrow K \pi, D^{0} \rightarrow K \pi \pi^{0}, D^{0} \rightarrow\right.$ $K \pi \pi \pi)$ and excluding $B^{0} \rightarrow D^{*-}(2010) K^{+}$contribution. Using Dalitz analysis we identified two resonant contributions: $\mathcal{B}\left(B^{0} \rightarrow \bar{D}^{0} K^{* 0}\right) \times$ $\mathcal{B}\left(K^{* 0} \rightarrow K^{+} \pi^{-}\right)=(3.9 \pm 0.6 \pm 0.4) \times 10^{-5}$ and $\mathcal{B}\left(B^{0} \rightarrow D_{2}^{*-}(2460) K^{+}\right) \times \mathcal{B}\left(D_{2}^{*-}(2460) \rightarrow\right.$ $\left.\bar{D}^{0} \pi^{-}\right)=(1.9 \pm 0.4 \pm 0.3) \times 10^{-5}$. We also set an 
upper limit at $90 \%$ CL on the CKM suppressed channel : $\mathcal{B}\left(B^{0} \rightarrow D^{0} K^{+} \pi^{-}\right)<1.9 \times 10^{-5}$. However, we come to the conclusion that measuring $\gamma$ is very difficult with this mode and that approximately $2000 \mathrm{fb}^{-1}$ are necessary to constrain $\gamma$ within $\pm 50^{\circ}$ at $3 \sigma$ level.

1.3. Search for $B \rightarrow D_{s}^{+} X_{\text {light }}$ with $X_{\text {light }} \equiv$ $\pi^{0}, a_{0}^{-}, a_{1}^{-}$

The value of $\sin (2 \beta+\gamma)$ can be extracted from the measurement of the time dependent $C P$ asymmetry in $B^{0} \rightarrow D^{-} X_{\text {light }}^{+}$decays where $X_{\text {light }}^{+} \equiv$ $\pi^{+}, a_{0}^{+}, a_{2}^{+}$. In this case, the asymmetry is given by : $\mathcal{A}_{C P}(\Delta t)=r \times \sin (2 \beta+\gamma) \times \sin \left(\Delta m_{d} \Delta t\right)$ where $r=\mathcal{B}\left(B^{0} \rightarrow D^{+} X_{\text {light }}^{-}\right) / \mathcal{B}\left(B^{0} \rightarrow\right.$ $\left.D^{-} X_{\text {light }}^{+}\right)$. The decay $B^{0} \rightarrow D^{+} X_{\text {light }}^{-}$is doubly Cabibbo suppressed and difficult to measure directly. Using $S U(3)$ flavor symmetry, it is possible to infer the value of $\mathcal{B}\left(B^{0} \rightarrow D^{+} X_{\text {light }}^{-}\right)$ from the value of $\mathcal{B}\left(B \rightarrow D_{s}^{+} X_{\text {light }}\right)$, the latter being less suppressed.

If $X_{\text {light }}^{+} \equiv \pi^{+}$, then $r$ is expected to be very small $(r \approx 0.02)$ which implies a small asymmetry. In this case $r$ may be deduced from the rate $\mathcal{B}\left(B^{+} \rightarrow D_{s}^{+} \pi^{0}\right)$. We measure this branching ratio from a sample of 124 millions of $B \bar{B}$ pairs, we do not see any significant signal and quote an upper limit at $90 \%$ CL of : $\mathcal{B}\left(B^{+} \rightarrow D_{s}^{+} \pi^{0}\right)<2.8 \times 10^{-5}$ in agreement with a previous measurement by CLEO $\left(<2.4 \times 10^{-4}\right.$ from ref. [2]) and with the value of $0.9 \times 10^{-5}$ expected from the rate of $\mathcal{B}\left(B^{0} \rightarrow D_{s}^{+} \pi^{-}\right)$ measured by Belle and BABAR experiments. If $X_{\text {light }}^{+} \equiv a_{0}^{+}\left(a_{2}^{+}\right), r$ might be quite large. This is due to the coupling constant of the $W$ to the $a_{0}$ scalar meson ( $a_{2}$ tensor meson) which is small and decreases the production rate of the Cabibbo allowed decay $B^{0} \rightarrow D^{-} a_{0}^{+}\left(a_{2}^{+}\right)$. The factorization hypothesis predicts a similar rate for Cabibbo allowed and Cabibbo suppressed decays [5] which results in $r \approx 1$. These decays are not yet within the experiment reach (branching ratios around $10^{-6}$ ), nevertheless, the theoretical predictions can be tested with the measurement of the branching ratio of the decay $B^{0} \rightarrow D_{s}^{+} a_{0}^{-}\left(a_{2}^{-}\right)$expected at larger values: $\mathcal{B}\left(B^{0} \rightarrow D_{s}^{+} a_{0}^{-}\left(a_{2}^{-}\right) \approx 7.5(1.5) \times 10^{-5}\right.$ (ref. $\left.[5,6]\right)$.
From a sample of 230 million of $B \bar{B}$ pairs, we measure these two branching ratios. The $a_{0}^{-}\left(a_{2}^{-}\right)$ is reconstructed in $a_{0(2)}^{-} \rightarrow \eta(\rightarrow \gamma \gamma) \pi^{+}$which has a branching ratio of the order of $100 \%$ (only $15 \%$ for the $a_{2}^{-}$). We do not find any significant signal and quote the upper limits at 90\% CL : $\mathcal{B}\left(B^{0} \rightarrow D_{s}^{+} a_{0(2)}^{-}\right)<4.0(25) \times 10^{-5}$ which shows a discrepancy of at least a factor two with the theoretical prediction for $a_{0}$.

\subsection{Charmless decays}

The decay $B^{+} \rightarrow K^{*+}\left(\rightarrow K^{+} \pi^{0}\right) \pi^{0}$ and its $C P$ asymmetry are particularly interesting in light of the recent measurement of direct $C P$ violation in the decay $B^{0} \rightarrow K^{+} \pi^{-}$[7]. It may provide valuable test of theoretical models such as those based on QCD factorization or SU(3) flavor symmetry. It has been argued that the influence of final state interactions like charming penguins and similar long distance rescattering effects on both the branching fraction and $C P$ asymmetry of $B \rightarrow K \pi$ decays may be significant. From a sample of 232 million of $B \bar{B}$ pairs we find $88.5 \pm 25.7$ signal events which correspond to the branching ratio : $\mathcal{B}\left(B^{+} \rightarrow K^{*+} \pi^{0}\right)=(6.9 \pm 2.0 \pm 1.3) \times 10^{-6}$ and we do not find any hint of direct $C P$ violation : $\mathcal{A}_{C P}=0.04 \pm 0.29 \pm 0.05$ [8]. These results do not rule out the charming penguins hypothesis considering the large values of the uncertainties for both the branching ratio and the $C P$ asymmetry.

\section{Selection of other recent analyses}

\subsection{Measurement of the $B^{0} \rightarrow D^{*-} D_{s}^{*+}$ and $D_{s}^{+} \rightarrow \phi \pi^{+}$branching ratios}

We present two measurements of the branching ratio $\mathcal{B}\left(B^{0} \rightarrow D^{*-} D_{s}^{*+}\right)$ which lead to a precise determination of the reference $\mathcal{B}\left(D_{s}^{+} \rightarrow \phi \pi^{+}\right)$. They have been performed on a sample of 123 million of $B \bar{B}$ pairs. The $B^{0} \rightarrow D^{*-} D_{s}^{*+} \rightarrow$ $\left(\bar{D}^{0} \pi^{-}\right)\left(D_{s}^{+} \gamma\right)$ decay is reconstructed using two different methods. The first one combines the fully reconstructed $D^{*-}$ with the photon from the $D_{s}^{*+} \rightarrow D_{s}^{+} \gamma$ decay, without explicit reconstruction of the $D_{s}^{+}$. To extract the num- 
ber of partially reconstructed events, we compute the "missing mass" $m_{\text {miss }}$ recoiling against the $D^{*-} \gamma$ system assuming that a $B^{0} \rightarrow D^{*-} D_{s}^{*+} \rightarrow$ $\left(\bar{D}^{0} \pi^{-}\right)\left(D_{s}^{+} \gamma\right)$ decay took place. For signal events, $m_{m i s s}$ peaks at the $D_{s}$ mass. We find, with this method, the following branching ratio : $\mathcal{B}_{1} \equiv \mathcal{B}\left(B^{0} \rightarrow D^{*-} D_{s}^{*+}\right)=(1.88 \pm 0.09 \pm$ $0.17) \%$ which is in agreement with the factorization model prediction : $\mathcal{B}\left(B^{0} \rightarrow D^{*-} D_{s}^{*+}\right)_{\text {theo }}=$ $(2.4 \pm 0.7) \%$. The second method uses a full reconstruction technique of the decay chain $B^{0} \rightarrow$ $D^{*-} D_{s}^{*+}$ where the $D_{s}$ candidate is reconstructed in the mode $: D_{s}^{+} \rightarrow \phi \pi^{+} \rightarrow\left(K^{+} K^{-}\right) \pi^{+}$. We measure the branching ratio $\mathcal{B}_{2} \equiv \mathcal{B}\left(B^{0} \rightarrow\right.$ $\left.D^{*-} D_{s}^{*+}\right) \times \mathcal{B}\left(D_{s}^{+} \rightarrow \phi \pi^{+}\right)=\left(8.81 \pm 0.86_{\text {stat }}\right) \times$ $10^{-4}$.

From the ratio $\mathcal{B}_{2} / \mathcal{B}_{1}$, where many systematics cancel out, we get a precise measurement of : $\mathcal{B}\left(D_{s}^{+} \rightarrow \phi \pi^{+}\right)=(4.81 \pm 0.52 \pm 0.38) \%$. [9]. which shows a different central value and an improvement on the uncertainty by about a factor of two compared to previous measurements [2].

2.2. Search for the rare decays $\bar{B}^{0} \rightarrow D^{(*) 0} \gamma$ Within the standard model, the rare decay $\bar{B}^{0} \rightarrow D^{(*) 0} \gamma$ is dominated by the W-boson exchange process. Its branching fraction is estimated to be of the order of $10^{-6}$ but the presence of a large $q \bar{q} \mathrm{~g}$ (color octet) component in the wave function of the $B$ meson may reduce the color suppression enough to enhance the branching fraction by a factor of 10 . A limit of $\mathcal{B}\left(\bar{B}^{0} \rightarrow D^{(*) 0} \gamma\right)<5.0 \times 10^{-5}$ at $90 \%$ CL has been published by the CLEO collaboration. With 87.8 million of $B \bar{B}$ pairs, we set an upper limit of $\mathcal{B}\left(\bar{B}^{0} \rightarrow D^{(*) 0} \gamma\right)<2.5 \times 10^{-5}$ at $90 \% \mathrm{CL}[10]$ in agreement with the theoretical expectations.

\subsection{Search for the rare decays $B^{+} \rightarrow$ $D^{(*)+} K^{0}$}

This decay is expected to occur via a pure annihilation diagram. Such processes provide interesting insights into the internal dynamics of $B$ mesons. This kind of diagram cannot be calculated in QCD factorization since both quarks play a role. The amplitudes are expected to be suppressed, with respect to the amplitudes of spectator quark trees, by a factor $f_{B} / m_{B} \approx 0.04$.
The branching fractions are expected to be of the order of $10^{-8}$ and have never been observed. Some studies [11] indicate, though, that processes with a spectator quark can contribute to annihilation-mediated decays by rescattering and the branching ratio is expected to raise up to $10^{-5}$ if large rescattering occurs [11]. We reconstruct the two decay modes $B^{+} \rightarrow D^{*+} K_{S}^{0}$ and $B^{+} \rightarrow D^{+} K_{S}^{0}$ within a sample of 226 million of $B \bar{B}$ pairs. We do not see any significant excess of signal, we therefore set the upper limits at $90 \% \mathrm{CL}: \mathcal{B}\left(B^{+} \rightarrow D^{+} K_{S}^{0}\right)<0.5 \times 10^{-5}$ and $\mathcal{B}\left(B^{+} \rightarrow D^{*+} K_{S}^{0}\right)<0.9 \times 10^{-5}$ thus beginning to constrain the rescattering effects.

\subsection{Search for the rare decays $B^{-} \rightarrow$ $D_{s}^{(*)-} \phi$}

In this other annihilation process $B^{-} \rightarrow$ $D_{s}^{(*)-} \phi$, the branching fraction is expected to be suppressed in the standard model down to $10^{-6}$ $10^{-7}$. Searches of $B^{-} \rightarrow D_{s}^{(*)-} \phi$ decays could be sensitive to the new physics (NP) contributions such as Higgs doublet model which predicts a branching fraction of the order of $10^{-5}$ or the minimal supersymmetric model with R-parity violation which predicts $10^{-4}$. Upper limits from CLEO are respectively 3.2 and $4.0 \times 10^{-4}$ at $90 \%$ CL. Based on 234 million of $B \bar{B}$ pairs, and reconstructing $D_{s}^{-}$into $\phi \pi^{-}$we have found no evidence for $B^{-} \rightarrow D_{s}^{(*)-} \phi$ decays. We set upper limits at $90 \% \mathrm{CL}$ for: $\mathcal{B}\left(B^{-} \rightarrow D_{s}^{-} \phi\right)<1.8 \times 10^{-6}$ and for $\mathcal{B}\left(B^{-} \rightarrow D_{s}^{*-} \phi\right)<1.1 \times 10^{-5} \quad$ [12] using the new $B A B A R \mathcal{B}\left(D_{s}^{-} \rightarrow \phi \pi^{-}\right)$value [section 2.1]. Our limits are more than two orders of magnitude lower than those of CLEO ruling out the two mentioned NP models.

\subsection{Search for $B \rightarrow J / \psi D$ Decays}

The spectra of the momentum of inclusive $J / \psi$ mesons in the $\Upsilon(4 S)$ rest frame observed by CLEO and by BABAR, compared with calculations using non-relativistic QCD (NRQCD), show an excess at low momentum, corresponding to a branching fraction of approximately $6 \times 10^{-4}$. Many hypotheses have been proposed to explain this result but no experimental evidence has been found to support them. The presence of $b \bar{u} c \bar{c}$ components (intrinsic charm) in 
the $B$-meson wave function has also been suggested to enhance the branching ratio of decays such as $B \rightarrow J / \psi \bar{D}(\pi)$ to the order of $10^{-4}$ while pertubative QCD predicts a branching ratio for $B \rightarrow J / \psi \bar{D}$ of $10^{-8}-10^{-9}$. We test the decay channels $B \rightarrow J / \psi D$ within a sample of 124 million of $B \bar{B}$ pairs. We do not find any evidence of signal and obtain upper limits of $1.3 \times 10^{-5}$ for $B^{0} \rightarrow J / \psi \bar{D}^{0}$ and $1.2 \times 10^{-4}$ for $B^{+} \rightarrow J / \psi D^{+}$at $90 \%$ CL. Therefore, intrinsic charm is ruled out as the explanation of low momentum $J / \psi$ excess in $B$ decays. More details on this analysis can be found in reference [13].

\subsection{Production and decay of the $\Xi_{c}^{0}$ and $\Omega_{c}^{0}$ at $B A B A R$}

We present a study of the $\Xi_{c}^{0}(c s d)$ [14], and $\Omega_{c}^{0}$ (ssc) [15] charmed baryons using for the former a luminosity of $116.1 \mathrm{fb}^{-1}$ through two decay modes : $\Xi_{c}^{0} \rightarrow \Omega^{-} K^{+}$and $\Xi_{c}^{0} \rightarrow \Xi^{-} \pi^{+}$. We measure, the ratio of the two decay rates to be $0.294 \pm 0.018 \pm 0.016$ which is compatible with the prediction, in a spectator quark model calculation, of 0.32 . For $\Omega_{c}^{0}$, we use $230 \mathrm{fb}^{-1}$ and we reconstruct the baryon through three decay modes to compare the branching fractions $[\mathcal{B} 1]$ : $\Omega_{c}^{0} \rightarrow \Omega^{-} \pi^{+},[\mathcal{B} 2]: \Omega_{c}^{0} \rightarrow \Omega^{-} \pi^{+} \pi^{-} \pi^{+}$and [B3] : $\Omega_{c}^{0} \rightarrow \Xi^{-} K^{-} \pi^{+} \pi^{+}$. We find the branching fraction ratios $[\mathcal{B} 3] /[\mathcal{B} 1]=0.31 \pm 0.015 \pm 0.040$ and $[\mathcal{B} 2] /[\mathcal{B} 1]<0.30$ at $90 \% \mathrm{CL}$. We also measure the $p^{*}$ distribution of both charmed baryons, in the $\Upsilon(4 S)$ frame, in order to study the production mechanisms in both $c \bar{c}$ and $B \bar{B}$ events. We find a double-peak structure in the $p^{*}$ spectrum of either baryon. This is due to two production mechanisms: the peak at lower $p^{*}$ is due to charmed baryon production in $B$ meson decays (first observation in the case of $\Omega_{c}^{0}$ ) and the peak at higher $p^{*}$ is due to charmed baryon production from the $c \bar{c}$ continuum. From these spectra we compute the cross-section of the production of $\Xi_{c}^{0}$ in continuum : $\sigma\left(e^{+} e^{-} \rightarrow c \bar{c} \rightarrow \Xi_{c}^{0} X\right) \times \mathcal{B}\left(\Xi_{c}^{0} \rightarrow\right.$ $\left.\Xi^{-} \pi^{+}\right)=(388 \pm 39 \pm 41) \mathrm{fb}$ and the rate of $\Xi_{c}^{0}$ production in $B$ decay : $\mathcal{B}\left(B \rightarrow \Xi_{c}^{0} X\right) \times \mathcal{B}\left(\Xi_{c}^{0} \rightarrow\right.$ $\left.\Xi^{-} \pi^{+}\right)=(2.11 \pm 0.19 \pm 0.25) \times 10^{-4}$.

The high rate of $\Xi_{c}^{0}$ production at low $p^{*}$ in $B$ decays (below $1.2 \mathrm{GeV} / \mathrm{c}$ ) implies that the in- variant mass of the recoiling antibaryon system is typically above $2.0 \mathrm{GeV} / c^{2}$. This can be explained naturally by a substantial rate of charmed baryon pair production through the $b \rightarrow c \bar{c} s$ weak decay process which was observed indirectly in a previous BABAR analysis [16].

\section{References}

\section{REFERENCES}

1. A. Giri, Y. Grossman, A. Soffer and J. Zupan, Phys. Rev. D 68, 054018 (2003).

2. S. Eidelman et al. [Particle Data Group], Phys. Lett. B 592, 1 (2004).

3. B. Aubert et al. [BABAR collaboration], hep-ex/0505084 and references therein.

4. R. Aleksan et al. Phys. Rev. D 67, 096002 (2003)

5. M. Diehl, G. Hiller, hep-ph/0105194

6. C.S. Kim, J.P. Lee, and M. Bauer, Z. Phys. C 29, 637 (1985).

7. B. Aubert et al. [BABAR collaboration], Phys. Rev. Lett. 93, 131801 (2004); Y. Chao et al. [Belle collaboration], Phys. Rev. Lett. 93, 191802 (2004).

8. B. Aubert et al. [BABAR collaboration], Phys. Rev. D 71, 111101(R) (2005)

9. B. Aubert et al. [BABAR collaboration], Phys. Rev. D 71, 091104 (2005) and references therein.

10. B. Aubert et al. [BABAR collaboration], hep-ex/0506070 and references therein. .

11. M. Blok, M. Gronau and J.L. Rosner, Phys. Rev. Lett. 78, 3999 (1997).

12. B. Aubert et al. [BABAR collaboration], hep-ex/0506073 and references therein.

13. B. Aubert et al. [BABAR collaboration], Phys. Rev. D 71, 091103 (2005) and references therein.

14. B. Aubert et al [BABAR collaboration], Phys. Rev. Lett. 95, 142003 (2005) and references therein.

15. B. Aubert et al [BABAR collaboration], hep-ex/0507011 and references therein.

16. B. Aubert et al. [BABAR collaboration], Phys. Rev. D 70, 091106 (2004). 